\title{
Important Parameters in Cytotoxicity Testing of Nanoparticles
}

\author{
C. MeINDL ${ }^{1,2}$, E. RoblegG ${ }^{3}$, T. R. PIEBeR ${ }^{2}$, E. FRÖHLICH ${ }^{1,2}$ \\ ${ }^{1}$ Center for Medical Research, Medical University of Graz, Austria \\ ${ }^{2}$ Department of Internal Medicine, Division of Endocrinology and Nuclear Medicine, Medical University of \\ Graz, Austria \\ ${ }^{3}$ Institute of Pharmaceutical Sciences, Department of Pharmaceutical Technology, Karl-Franzens-University \\ of Graz, Austria \\ E-mail: eleonore.froehlich@medunigraz.at (E. Fröhlich)
}

Sci Pharm. 2010; 78: 575

doi:10.3797/scipharm.cespt.8.LNT01

Background: The use of nano-sized materials (NMs) offers exciting new options in technical and medical applications. Adverse effects on cells and organs, however, have also been reported. It is known that NMs may interfere with conventional assays. It may be suspected that inter-cell line differences in the sensitivity to NMs also exist. NMs may also interact physicochemically with plasma membranes. Cells in suspension, where a relatively large area is exposed, may therefore react different from adherent cells. As NMs by interaction with the replicative machinery may inhibit proliferation, also the proliferation rate (length of the doubling time) may be an important parameter. Methods: To assess the effect of the medium 20-1000 nm large carboxyl polystyrene particles (CPS) were tested in the presence of different amounts of fetal bovine serum. Potential interference was evaluated by testing in the cytotoxicity screening assays WST-1, MTT, MTS, Neutral Red, Sulforhodamin $B$, leucine incorporation and ATP content. IC50 concentrations were compared between 20 different cell lines.

Results: The size of small $(20-60 \mathrm{~nm})$ CPS increased markedly from medium with $0 \%$ to $10 \%$ FBS; for larger CPS only minimal differences were seen. $20 \mathrm{~nm}$ CPS in medium with 0\% FBS acted cytotoxic in all cell lines. $20 \mathrm{~nm}$ CPS in $5 \%$ and $10 \%$ FBS and larger CPS in all media were only minimally cytotoxic to nonphagocytic cells. In phagocytic cells also CPS of $\geq 500 \mathrm{~nm}$ acted cytotoxic. Half maximal inhibition concentrations (IC50) in a given cell line did not differ markedly between the screening assays but varied more than 10 times between the cell lines. IC50 values were significantly lower in suspension cells. Large cells and human cell lines with long doubling times were more resistant to the cytotoxic action of $20 \mathrm{~nm}$ CPS.

Conclusions: In addition to medium composition, growth characteristics, proliferation and cell size of the cell line used for testing may influence the IC50 values of NMs. Cells in suspension are especially sensitive to the cytotoxic action of small carboxyl polystyrene nanoparticles.

This work was supported by the FP6 European integrated project "NanoBioPharmaceutics", NMP4-CT-2006-026723 and by the RPC project 'Nano-structured Materials for Drug Targeting, Release and Imaging'. 